\title{
Study of the hadronic photon structure function with the DELPHI detector at LEP2.
}

\author{
I. Tyapkin, N. Zimine, A. Zinchenko
}

\begin{abstract}
The photon structure function $F_{2}^{\gamma}$ has been studied at average $Q^{2}$ values of $19 \mathrm{GeV}^{2}$, $40 \mathrm{GeV}^{2}$ and $101 \mathrm{GeV}^{2}$. The data correspond to the integrated luminosity of 548 $\mathrm{pb}^{-1}$, collected by the DELPHI detector during the 1998-2000 LEP runs. Experimental distributions are compared with predictions of different models. The procedure of $F_{2}^{\gamma}$ extraction from the data and its uncertainty are discussed. The $F_{2}^{\gamma}$ estimated from the fit of models to the data and compared with theoretical expectations based on different models. A result for $Q^{2}$ evolution of the photon structure function has been obtained.
\end{abstract}




\section{Introduction}

The photon structure function has been measured at PETRA and PEP $[1,2,3,4,5,6]$ and more recently at TRISTAN $[7,8]$ and LEP $[9,10,11,12,13,14,15,16,17,18]$ in the reaction $e^{+} e^{-} \rightarrow e^{+} e^{-} X$, where $X$ is a multihadronic system and when one of the scattered leptons is observed at a large scattering angle (tagging condition) while the other, remaining at a small angle, is undetected (anti-tagging condition). This reaction can be described as a deep inelastic $e \gamma$ scattering (DIS), where $\gamma$ is almost a real photon. The corresponding cross-section is usually expressed in terms of the photon structure functions $F_{2}^{\gamma}\left(x, Q^{2}\right)$ and $F_{L}\left(x, Q^{2}\right)$ :

$$
\frac{d \sigma}{d E_{t a g} d \cos \left(\theta_{t a g}\right)}=\frac{4 \pi \alpha^{2} E_{t a g}}{Q^{4} y}\left[\left(1+(1-y)^{2}\right) F_{2}^{\gamma}\left(x, Q^{2}\right)-y^{2} F_{L}\left(x, Q^{2}\right)\right] .
$$

Here, $E_{t a g}$ and $\theta_{t a g}$ are the energy and polar angle of the tagged lepton, $Q^{2}=4 E_{\text {tag }} E_{\text {beam }} \sin ^{2}\left(\theta_{\text {tag }} / 2\right), \mathrm{x}=Q^{2} /\left(Q^{2}+W^{2}+P^{2}\right), \mathrm{y}=1-\left(E_{\text {tag }} / E_{\text {beam }}\right) \cos ^{2} \theta_{\text {tag }}, \mathrm{W}$ is the invariant mass of the hadronic system and $P^{2}$ is the negative four-momentum squared for the virtual photon emitted from the anti-tagged electron. Anti-tagging conditions ensures that $P^{2}$ is much smaller than $Q^{2}$. Nevertheless the influence of $P^{2}$ is not negligible and should be taken into account in the final result. Due to small values of $y$ in the experimentally accessible region, an influence of $F_{L}$ on the cross-section is small (about few percent) and may be taken into account in the simplified way given an additional uncertainty of these measurements.

The contributions to the photon structure function mostly come from the diagrams in Fig.1. The point-like contribution (Fig.1a) can be evaluated in the frame of perturbative quantum chromodynamics (QCD). The contribution from the non-perturbative

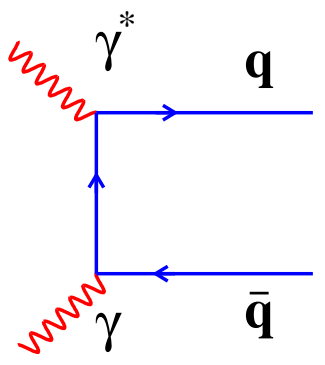

$\mathbf{a}$

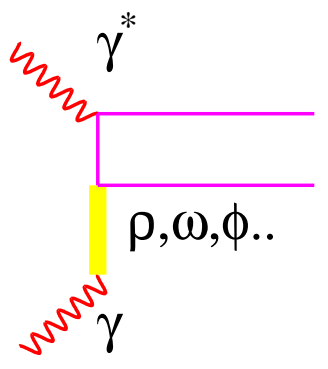

b

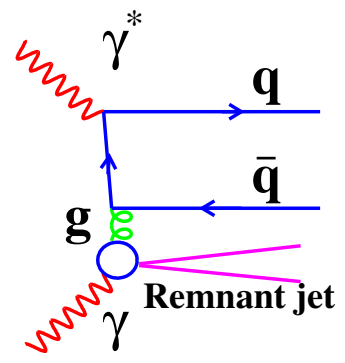

c

Figure 1: The point-like (a), the hadron-like (b) and RPC (c) contributions to $F_{2}^{\gamma}$.

component (Fig.1b VDM-like) is significant and can not be calculated in the frame of perturbative theory. In 1996 OPAL [19] and DELPHI [20] have stressed the importance of a proper description of the hadronic state by models used in the analysis. It has been shown, that a poor description of the hadronic system produced in $\gamma \gamma^{*}$ interactions can be due to a missing component in the model. Moreover, problems in the description of the hadronic system leads to a distortion of a correlation between $x_{v i s}$ and $x_{\text {true }}$ given by the model. Both factors mentioned above can result in a significant bias in an estimation of the structure function. All studies before 1996 were based on the generators 
included two components, the QPM-like describing the perturbative part (Fig. 1a), and the VDM-like for the hadron-like part (Fig. 1b). As shown by the DELPHI collaboration in [20] this approach gives a poor description of the final state and, as a result, leads to significant bias in the structure function estimation. The solution proposed [20] consists in introducing the hard scattering process (Fig.1c) in the description of the process (RPCresolved photon contribution). Here different partonic densities of the photon can be used to describe the interaction between highly virtual photon and one of the partons of the resolved photon. Such a modification significantly improves the agreement of simulated events with experimental data. This leads to a more correct description of the final state topology which is crucial for the interpretation of the results. This approach can be realised by making use of the TWOGAM [21] and PHOJET [27] generators. Recently, the general-purpose generators PYTHIA [29] and HERWIG [30] have also been adopted to DIS study. All new generators use the parton density functions of the photon which are obtained under recent theoretical assumptions but require additional input data, namely, experimental measurements of the photon structure function. Influence of the parton density functions on the results given by TWOGAM and PHOJET is much smaller because these generators use it only for RPC. From this point of view, new measurements will definitely affect theoretical predictions. Several authors [22, 23, 24, 25, 26] tried to calculate corresponding $F_{2}^{\gamma}$ but the difference in their results, originated from the treatment of hadronic part and choice of boundary conditions for the perturbative equations is rather large. That is why new studies are needed to improve an understanding of the real photon which will be increasingly important in $\gamma \gamma$ and $\gamma$ p interactions at higher energies.

\section{Event selection}

The detailed description of the DELPHI detector can be found in [31]. The components of the detector relevant to the analysis of $\gamma \gamma$ events have been described in our previous papers [10], [32]. Data used in this analysis were collected with the DELPHI detector at LEP $e^{+} e^{-}$collider during the 1998-2000 runs. The range of centre-of-mass energies is from $188 \mathrm{GeV}$ to $208 \mathrm{GeV}$. The tagged particles were detected by the DELPHI luminometer STIC (Small angle Tail Calorimeter).

The following criteria were used to select a pure sample of $\gamma \gamma^{*}$ events:

1. The energy deposited by the tagged electron (or positron) must be greater than $0.4 * E_{\text {beam }}$ (tagging requirement);

2. No additional clusters with energy exceeding $0.3 * E_{\text {beam }}$ must be observed anywhere in the forward calorimeters (anti-tagging requirement);

3. The track multiplicity is 3 or more. This includes only tracks with momenta greater than $0.25 \mathrm{GeV} / c$ with a polar angle between $20^{\circ}$ and $160^{\circ}$ and an impact parameter less than $4 \mathrm{~cm}$ in the radial direction and less than $8 \mathrm{~cm}$ along the beam (hadronic final state selection);

4. The visible invariant mass of the hadronic system must be greater than $2.5 \mathrm{GeV}$;

5. The vector sum of transverse momenta of all particles, including tagged particle, normalised to $E_{\text {beam }}$ must be less than 0.12 ; 
6. The absolute value of the sum of longitudinal momenta of particles, including tagged particle, normalised to $E_{\text {beam }}$ must be greater than 0.6.

In this analysis all the DELPHI calorimeters are used for event reconstruction. It strengthens the correlation between $x_{\text {true }}$ and $x_{v i s i b l e}$. Calorimetric clusters were accepted if their energy deposition exceeded $0.5 \mathrm{GeV}$ in the forward or barrel electromagnetic calorimeters, $1.0 \mathrm{GeV}$ in the hadron calorimeter and $1.5 \mathrm{GeV}$ in the luminometer.

Finally, a total of 24222 events were selected which corresponds to visible cross-section of $44.2 \mathrm{pb}$. The background from $\gamma \gamma^{*} \rightarrow \tau \tau$ and $\gamma \gamma^{*} \rightarrow e^{+} e^{-}$interactions was estimated from a simulation as $1.6 \mathrm{pb}$ and $0.6 \mathrm{pb}$ respectively. The background from $Z^{0} \gamma$ hadronic decays was estimated as $0.5 \mathrm{pb}$. In the interaction $\gamma \gamma \rightarrow$ hadrons high energy decay products can produce clusters in the STIC above tagging threshold and mimic the tagging particle. Such contribution estimated from a simulation as $0.7 \mathrm{pb}$. The contamination from other sources of background was found to be much lower. After subtraction of the background the visible cross-section of the investigated process was estimated as being $40.8 \mathrm{pb}$. This sample was used as a basis of this study, some variations of the selection criteria were allowed to study the systematics. The average $Q^{2}$ for the selected events is about $40 \mathrm{GeV}^{2}$. The trigger efficiency was studied and found to be of the order of $98 \pm 1 \%$.

\section{Event Generators}

Three generators were used to produce simulated samples. A two-photon event generator TWOGAM [21] was successfully tested in previous DELPHI studies. The total crosssection is described by the sum of three parts: the point-like (QPM) component, resolved photon contribution (RPC) and soft hadronic (VDM) component. The QPM part based on the exact decomposition of the matrix element of the process and the exact differential cross-sections from [33] are used. The quark masses are taken to be $0.3 \mathrm{GeV}$ for $u$ and $d$ quarks, $0.5 \mathrm{GeV}$ for $s$ and $1.6 \mathrm{GeV}^{2}$ for $c$ quarks. For the RPC perturbative part the lowest order cross-sections are used. Only the transverse-transverse part of the luminosity function is used in this case. There is no initial or final state parton showering. Strings are formed following the colour flow of the sub-processes. The remnant of a quark is an antiquark (and vice versa), and the remnant of a gluon is a $q \bar{q}$ pair. The produced system is fragmented as a string by JETSET 7.4 [29].

The Gordon-Storrow [23] parameterization were used in this analysis. A transverse momentum cutoff, $p_{t}^{c u t}=1.8 \mathrm{GeV}$, is applied to the partons of the resolved photons to separate soft from hard processes. In this analysis the GVDM structure function multiplied by the factor $\left(1-x_{\text {true }}\right)$ for the soft hadronic part was used. TWOGAM treats exactly the kinematics of the scattered electron and positron, and uses exact (unfactorised) expressions for the two photon luminosity function. New version of TWOGAM (2.04) was used in this analysis.

Second Monte Carlo event generator used in this analysis is PHOJET [27] (version 1.12). The generator includes the exact photon flux simulation for photon-photon processes in lepton-lepton collisions. The ideas and methods used in the program are based mainly on the Dual Parton Model (DPM). In order to combine the DPM which describes soft processes with the predictive power of perturbative QCD, the event generator is formulated as a two-component model (soft and hard components). On the basis of 
the optical theorem, Regge phenomenology is used to parametrize the total and elastic cross-sections as well as a series of partial inelastic cross-sections. In order to conserve s - channel unitarity, Gribov's Reggeon calculus is applied. Consequently, the model predicts so-called "multiple parton interactions" in one event. Since the unitarization of soft and hard processes is treated in unified way, multiple soft and hard interactions may be generated in one event. Hard scattering processes are simulated using lowest-order perturbative QCD. Initial state and final state parton showers are generated in leading-log

approximation. Some coherence effects (angular ordering in the emissions) are taken into account. For the fragmentation of parton configurations, the JETSET 7.4 program is also used. The generator includes the exact photon flux simulation for photon-photon processes in lepton-lepton collisions. The GRV [24] parameterization were used in this analysis. A transverse momentum cutoff, $p_{t}^{c u t}=2.5 \mathrm{GeV}$, is applied to the partons of the resolved photons to separate soft from hard processes. Unfortunately, program can run only in the invariant mass region above $5 \mathrm{GeV}$.

Third Monte Carlo program used in this analysis is the PYTHIA (version 6.143) general purpose event generator. In this model different kinds of events distinguished as: direct events, VDM events and anomalous events [28]. In order that the above classification is smooth and free of double counting the cutoff parameters was introduced on the lavel of real photon fluctuation $\gamma \rightarrow q \bar{q}$ and the final hadronic system creation $\gamma \gamma^{*} \rightarrow q \bar{q}$. The VDM and anomalous events together are called resolved ones. But, these two classes differ in the structure of underlying event and in the appearance of soft events. The superposition of events mentioned above applies separately for each of the two incoming photons and forms six distinct classes of events: direct-direct, VDM-VDM, anomalous-anomalous, direct-VDM, direct-anomalous and VDM-anomalous. In the case of DIS only one of the photons can be resolved and only direct-direct, direct-VDM and direct-anomalous components should be taken in to account.

only direct-direct, direct-VDM and direct-anomalous components should be taken into account. These three contributions are similar to the TWOGAM and PHOJET classification. All generated samples were processed by the full detector simulation and reconstruction programs.

\section{Comparison of experimental and simulated data}

As it was mentioned in Section 1 a good modelling is necessary for accurate measurements of $F_{2}^{\gamma}$. Due to finite detector resolution and acceptance, the event final state topology effects the correlation between $x_{\text {true }}$ and $x_{\text {visible }}$ and the acceptance factor which determines the transition from $x_{\text {true }}$ distribution to $F_{2}^{\gamma}$. Each component of the model has different $x_{\text {true }}-x_{\text {visible }}$ correlation and the acceptance factor. Therefore, not only the final state topology for each component but also the cross-section of each component should be properly simulated. To check available models the inclusive and global event distributions should be compared for data and Monte Carlo predictions. Special attention should be paid to the distributions which are not strongly correlated with $x_{\text {true }}$ and cannot be corrected by tuning of $F_{2}^{\gamma}$.

All three generators mentioned above were used to describe experimental distributions. The $Q^{2}, E_{\text {tag }} / E_{\text {beam }}$ and $W_{\text {vis }}$ spectra are shown in Fig.2. Due to the limitations in the PHOJET and PYTHIA only events with invariant mass above 5 Gev are selected for these 
plots. In this invariant mass region PHOJET giving best description of the experimental data. One can see that TWOGAM overestimates and PHOJET and essentially PYTHIA underestimates the total visible cross-section. All models fail to describe low $\mathrm{W}$ region. A detailed analysis of these models shows that TWOGAM and PHOJET differ mostly in their point-like part due to the $p_{t}=2.5 \mathrm{GeV}$ cut applied in PHOJET to the partons in the final state. The observed $x$ distributions in different $Q^{2}$ regions are shown in Fig3. Here again PHOJET have the best agreement with data in all $Q^{2}$ regions. The energy flow versus pseudorapidity defined as $\eta=-\ln (\tan (\theta / 2))$, where $\theta$ is the polar angle of final state particles, is shown in Fig4. All models used in this analysis (essentially the PHOJET and PYTHIA) fail to describe the energy flow in the central region. This indicate some inconsistency in the description of final state topology and will increase uncertainty in the extracted $F_{2}^{\gamma}$.

\section{Extraction of the structure function}

The usual way of $F_{2}^{\gamma}$ extraction from data is to use the method of regularized unfolding (for example, Blobel's unfolding program [37]). In this method a Monte Carlo simulation is used to find a response matrix for a transition from $x_{\text {true }}$ to $x_{\text {visible }}$ which includes the effect of limited detector resolution ( $W_{\text {visible }}$ is lower than $W_{\text {true }}$ ). Then a regularized unfolding obtains the $x_{\text {true }}$ distribution for the data. As a last step $F_{2}^{\gamma}$ is determined by reweighting the input structure function of the Monte Carlo according to the ratio of the unfolded $x_{\text {true }}$ distribution to the $x_{\text {true }}$ distribution in the Monte Carlo. This step takes into account the efficiency effect (not all the events can be detected, triggered or selected by the selection criteria). Thus, the unfolding method does not take into account a difference in the correlations for each of the components in the model. The reweighting factor is also determined by unfolding for a sum of the components in the model without taking into account their differences. These two factors make usual unfolding procedure not adequate to the task. A priori some of the components can be correctly simulated in the generator but some others should be fitted to the data. Only on a basis of statistical comparison of many of reweighted simulated and experimental distributions one can decide which components should be modified.

In this analysis the MINUIT program is used. The correction factor $A_{i j}$ are introduced for each bin of $x_{\text {true }}^{i}$ (here $i$ running form 1 to 4 ) and each model component (the QPM $\mathrm{j}=1, \mathrm{VDM} \mathrm{j}=2$ and $\mathrm{RPC} \mathrm{j}=3$ ). These factors $A_{i j}$ are the free parameters for the MINUIT fit of $x_{\text {visible }}$ Monte Carlo distribution to the date one. In each fit only four correction factors $A_{1 k}, A_{2 l}, A_{3 m}$ and $A_{4 n}$ (where $k, l, m, n$ can be any number from 1 to 3 ) are used. Thus, the total 81 combinations are considered for four $x_{\text {true }}$ bins analysis. As a result, we have a set of structure functions extracted from the data with corresponding reweighted simulated distributions. Most of the fits give the statistically acceptable quality and only on the basis of the analysis of many additional distributions one can make a choice between these fits. The statistical analysis of these distributions gives $\chi^{2}$, which is then considered as a weight factor for $F_{2}^{\gamma}$ measurement in each fit.

The difference in each $x$ bin for different fits represents the systematic error due to the choice of the model or combinations of the models for the fit. Even the statistical error for each $x$ bin depends on the choice of the model due to different efficiencies of event selection for each model. Thus, only combination of all fits gives the final result. 
The test of the fitting procedure has been done. The sample simulated by the PHOJET program was used to extract the structure function used in TWOGAM. The results of this test together with the test of Blobel's unfolding program are shown in Fig.5(a,b). Solid lines are the structure function used in the TWOGAM. Open circles correspond to the fit with the Monte Carlo sample treated as a sum of three components. Black dots in Fig.5a better represent structure function and correspond to the complete MINUIT fitting procedure described above. For comparison the results of unfolding procedure are presented in Fig.5b. As expected, the unfolding procedure leads to some bias and underestimates the errors.

The value of $F_{L}^{\gamma}$ was estimated by TWOGAM and then subtracted from the result. The same generator have been used to estimate correction factors for each $\mathrm{x}$-bin which taking into account the non-zero virtuality of the target photon in the experiment. All results then corrected on these factors to get $F_{2}^{\gamma}$ for the $P^{2}=0$. Full event sample have been used to extract $F_{2}^{\gamma}$ with average $<Q^{2}>=40 \mathrm{GeV}^{2}$. Structure function in the low $Q^{2}$ region $\left(<Q^{2}>=19 \mathrm{GeV}^{2}\right)$ have been extracted from the subsample of events with $5<Q^{2}<30 \mathrm{GeV}^{2}$. Selecting subsample with $60<Q^{2}<260 \mathrm{GeV}^{2}$ structure function in the high $Q^{2}$ region $\left(<Q^{2}>=101 \mathrm{GeV}^{2}\right)$ was extracted. Results of the $F_{2}^{\gamma}$ extraction with the use of different models are presented in Fig6-8 and in the Tables 1-3.

\begin{tabular}{|c|c|c|c|c|c|c|c|c|c|}
\hline Model & $x$ range & $F_{2}^{\gamma} / \alpha$ & $\begin{array}{c}\text { Stat. } \\
\text { err. }\end{array}$ & $\begin{array}{c}\text { Mod. } \\
\text { err. }\end{array}$ & $\begin{array}{c}E_{\text {flow }} \\
\text { err. }\end{array}$ & $\begin{array}{c}\text { Det. } \\
\text { err. }\end{array}$ & $\begin{array}{c}\text { Back. } \\
\text { err. }\end{array}$ & $\begin{array}{c}\text { Tot. Sys. } \\
\text { err. }\end{array}$ & Tot. err. \\
\hline 1 & $0.001-0.02$ & 0.490 & 0.015 & 0.028 & 0.022 & 0.018 & 0.004 & 0.040 & 0.043 \\
2 & & 0.425 & 0.013 & 0.026 & 0.027 & 0.018 & 0.004 & 0.042 & 0.044 \\
3 & & 0.399 & 0.020 & 0.038 & 0.025 & 0.018 & 0.004 & 0.049 & 0.053 \\
\hline 1 & $0.02-0.1$ & 0.311 & 0.010 & 0.007 & 0.019 & 0.010 & 0.002 & 0.023 & 0.025 \\
2 & & 0.306 & 0.010 & 0.005 & 0.080 & 0.010 & 0.002 & 0.081 & 0.081 \\
3 & & 0.334 & 0.025 & 0.015 & 0.040 & 0.010 & 0.002 & 0.044 & 0.051 \\
\hline 1 & $0.001-0.1$ & 0.336 & 0.011 & 0.025 & 0.017 & 0.015 & 0.003 & 0.034 & 0.036 \\
2 & & 0.321 & 0.010 & 0.024 & 0.070 & 0.015 & 0.003 & 0.075 & 0.076 \\
3 & & 0.352 & 0.024 & 0.035 & 0.035 & 0.015 & 0.003 & 0.052 & 0.057 \\
\hline 1 & $0.1-0.3$ & 0.328 & 0.008 & 0.010 & 0.017 & 0.010 & 0.003 & 0.022 & 0.024 \\
2 & & 0.317 & 0.010 & 0.012 & 0.050 & 0.010 & 0.003 & 0.052 & 0.054 \\
3 & & 0.235 & 0.012 & 0.020 & 0.025 & 0.010 & 0.003 & 0.034 & 0.036 \\
\hline 1 & $0.3-0.8$ & 0.374 & 0.028 & 0.015 & 0.019 & 0.013 & 0.003 & 0.028 & 0.039 \\
2 & $0.3-0.5$ & 0.309 & 0.050 & 0.040 & 0.060 & 0.013 & 0.003 & 0.073 & 0.088 \\
3 & $0.3-0.5$ & 0.341 & 0.055 & 0.065 & 0.040 & 0.013 & 0.003 & 0.077 & 0.096 \\
\hline
\end{tabular}

Table 1. Summary for the $F_{2}^{\gamma}$ estimated by the TWOGAM(1), PHOJET(2) and PYTHIA(3) generated data for the sample with average $<Q>^{2}=19 \mathrm{GeV}^{2}$. 


\begin{tabular}{|c|c|c|c|c|c|c|c|c|c|}
\hline Model & $x$ range & $F_{2}^{\gamma} / \alpha$ & $\begin{array}{c}\text { Stat. } \\
\text { err. }\end{array}$ & $\begin{array}{c}\text { Mod. } \\
\text { err. }\end{array}$ & $\begin{array}{c}E_{\text {flow }} \\
\text { err. }\end{array}$ & $\begin{array}{c}\text { Det. } \\
\text { err. }\end{array}$ & $\begin{array}{c}\text { Back. } \\
\text { err. }\end{array}$ & $\begin{array}{c}\text { Tot. Sys. } \\
\text { err. }\end{array}$ & Tot. err. \\
\hline 1 & $0.001-0.02$ & 0.655 & 0.010 & 0.029 & 0.035 & 0.023 & 0.004 & 0.047 & 0.048 \\
2 & & 0.630 & 0.015 & 0.020 & 0.024 & 0.025 & 0.004 & 0.040 & 0.043 \\
3 & & 0.610 & 0.020 & 0.047 & 0.045 & 0.025 & 0.004 & 0.069 & 0.073 \\
\hline 1 & $0.02-0.1$ & 0.415 & 0.007 & 0.022 & 0.015 & 0.008 & 0.002 & 0.020 & 0.021 \\
2 & & 0.384 & 0.012 & 0.014 & 0.048 & 0.012 & 0.002 & 0.051 & 0.053 \\
3 & & 0.485 & 0.015 & 0.025 & 0.025 & 0.012 & 0.002 & 0.037 & 0.040 \\
\hline 1 & $0.001-0.1$ & 0.441 & 0.008 & 0.020 & 0.018 & 0.007 & 0.002 & 0.028 & 0.029 \\
2 & & 0.418 & 0.012 & 0.018 & 0.041 & 0.010 & 0.002 & 0.046 & 0.048 \\
3 & & 0.500 & 0.016 & 0.035 & 0.032 & 0.010 & 0.002 & 0.049 & 0.052 \\
\hline 1 & $0.1-0.3$ & 0.438 & 0.022 & 0.034 & 0.013 & 0.012 & 0.004 & 0.022 & 0.025 \\
2 & & 0.325 & 0.018 & 0.020 & 0.032 & 0.013 & 0.004 & 0.040 & 0.044 \\
3 & & 0.290 & 0.015 & 0.045 & 0.030 & 0.013 & 0.004 & 0.056 & 0.058 \\
\hline 1 & $0.3-0.8$ & 0.501 & 0.017 & 0.020 & 0.012 & 0.013 & 0.005 & 0.027 & 0.031 \\
2 & & 0.590 & 0.024 & 0.025 & 0.080 & 0.015 & 0.005 & 0.085 & 0.089 \\
3 & & 0.371 & 0.022 & 0.030 & 0.085 & 0.015 & 0.005 & 0.092 & 0.094 \\
\hline \hline
\end{tabular}

Table 2. Summary for the $F_{2}^{\gamma}$ estimated by the TWOGAM(1), PHOJET(2) and PYTHIA(3) generated data for the full sample with average

$$
<Q^{2}>=\mathbf{4 0} \mathrm{GeV}^{2} \text {. }
$$

\begin{tabular}{|c|c|c|c|c|c|c|c|c|c|}
\hline Model & $x$ range & $F_{2}^{\gamma} / \alpha$ & $\begin{array}{c}\text { Stat. } \\
\text { err. }\end{array}$ & $\begin{array}{c}\text { Mod. } \\
\text { err. }\end{array}$ & $\begin{array}{c}E_{\text {flow }} \\
\text { err. }\end{array}$ & $\begin{array}{c}\text { Det. } \\
\text { err. }\end{array}$ & $\begin{array}{c}\text { Back. } \\
\text { err. }\end{array}$ & $\begin{array}{c}\text { Tot. Sys. } \\
\text { err. }\end{array}$ & Tot. err. \\
\hline 1 & $0.001-0.02$ & 0.885 & 0.101 & 0.106 & 0.035 & 0.023 & 0.005 & 0.117 & 0.154 \\
2 & & 0.939 & 0.076 & 0.139 & 0.024 & 0.025 & 0.005 & 0.143 & 0.162 \\
3 & & 0.965 & 0.065 & 0.140 & 0.045 & 0.025 & 0.005 & 0.149 & 0.163 \\
\hline 1 & $0.02-0.1$ & 0.530 & 0.031 & 0.022 & 0.015 & 0.007 & 0.002 & 0.028 & 0.042 \\
2 & & 0.563 & 0.020 & 0.015 & 0.048 & 0.010 & 0.002 & 0.051 & 0.055 \\
3 & & 0.663 & 0.018 & 0.025 & 0.035 & 0.010 & 0.002 & 0.044 & 0.047 \\
\hline 1 & $0.001-0.1$ & 0.587 & 0.032 & 0.020 & 0.013 & 0.006 & 0.002 & 0.025 & 0.041 \\
2 & & 0.569 & 0.021 & 0.018 & 0.040 & 0.009 & 0.002 & 0.045 & 0.050 \\
3 & & 0.668 & 0.019 & 0.023 & 0.033 & 0.009 & 0.002 & 0.041 & 0.045 \\
\hline 1 & $0.1-0.3$ & 0.492 & 0.023 & 0.015 & 0.012 & 0.013 & 0.004 & 0.024 & 0.033 \\
2 & & 0.326 & 0.016 & 0.030 & 0.032 & 0.014 & 0.004 & 0.046 & 0.049 \\
3 & & 0.458 & 0.015 & 0.036 & 0.030 & 0.014 & 0.004 & 0.049 & 0.051 \\
\hline 1 & $0.3-0.8$ & 0.648 & 0.029 & 0.020 & 0.012 & 0.013 & 0.005 & 0.027 & 0.040 \\
2 & & 0.670 & 0.030 & 0.024 & 0.080 & 0.015 & 0.005 & 0.085 & 0.090 \\
3 & & 0.793 & 0.025 & 0.030 & 0.085 & 0.015 & 0.005 & 0.091 & 0.098 \\
\hline \hline
\end{tabular}

Table 3. Summary for the $F_{2}^{\gamma}$ estimated by the TWOGAM(1), PHOJET(2) and PYTHIA(3) generated data for the sample with average < $Q^{2}>=101 \mathrm{GeV}^{2}$.

Bins in $x$ are chosen to have compatible statistics in each of them and correlations between bins to be not too high. For all $Q^{2}$ regions the same $x$-bins are used 0.001-0.02, 0.02-0.1, 0.1-0.3, and 0.3-0.8. The first error is the statistical one (Stat. err.) which in this approach also depends on the model, and in some sense, carries some systematic uncertainty. The model dependent shift in $F_{2}^{\gamma}$ measured in each $x$ bin was interpreted as a modelling systematics (Mod. err.) and is shown in the Table 1-3 in the fifth column. 
Fit brings to the good agreement between most of distributions in data and Monte Carlo but it does not help in improving an agreement in the energy flow distribution. It is why systematic error due to the uncertainty in the energy flow description was studied separately. As it is seen from the Monte Carlo study correction to the $E_{\text {flow }}$ leads to the shift of the extracted value of $F_{2}^{\gamma}$. This shift interpreted as a systematic error $\left(E_{\text {flow }}\right)$. The shift of unfolding results due to variation in the selection criteria $\left(W_{\min }, N_{\min }^{\operatorname{trk} \ldots)}\right.$, thresholds for detection of neutrals by the calorimeters and uncertainty in the measurement of invariant mass was interpreted as a detector dependent systematic error (Det. err.). The background systematic error (Back. err.) reflects an uncertainty in the knowledge of the background and was estimated from $Z^{0} \gamma$ hadronic and $\gamma \gamma \rightarrow \tau \tau$ Monte Carlo as an uncertainty with which we are able to describe the corresponding data samples. Certainly, there are some other sources of systematics in the measurements, but their influence is estimated as much lower. The correlation matrix for each of the presented results was checked. The maximum correlation between bins is found to be below 0.40 . Typical values for the correction factors in the fit with the best $\chi^{2}$ are from 0.8 to 1.2 .

Conclusions drawn from the results in the Table 1-3 are the following: 1 . Taking into account a total error all generators give consistent results. 2. The main contribution to the total error comes from the model dependent systematics and poor simulation of the event final state topology. The systematic errors for the results extracted with the use of PHOJET and PYTHIA are larger than the errors in case of TWOGAM mostly due to the higher disagreement between data and Monte Carlo predictions for these generators in the description of the event topology. 3. In all three $Q^{2}$ regions results are higher than the GRV and SaS models predictions and consistent with TWOGAM prediction value of structure function in the lowest $x$ bin. At the same time, in the $x$ region larger than 0.02 results are systematically lower than TWOGAM prediction and close to the GRV model in the $x$ region above 0.1 .

To study the $Q^{2}$ evolution of $F_{2}^{\gamma}$, results from the Table 1-3 taken with the use of TWOGAM generator were used. The $x$ intervals $0.001-0.02,0.001-0.1$ and $0.3-0.8$ are chosen. The structure functions were extracted for three $Q^{2}$ intervals $10-30 \mathrm{GeV}^{2}, 30$ $60 \mathrm{GeV}^{2}$ and $60-260 \mathrm{GeV}^{2}$. Only results extracted with TWOGAM are presented for evolution study. The results for low and high $Q^{2}$ bins can be seen in the Tables 1-3 and for the intermediate $Q^{2}$ bin are shown in Table 4 .

\begin{tabular}{|c|c|c|c|c|c|c|c|c|}
\hline$x$ range & $F_{2}^{\gamma} / \alpha$ & $\begin{array}{c}\text { Stat. } \\
\text { err. }\end{array}$ & $\begin{array}{c}\text { Mod. } \\
\text { err. }\end{array}$ & $\begin{array}{c}E_{\text {flow }} \\
\text { err. }\end{array}$ & $\begin{array}{c}\text { Det. } \\
\text { err. }\end{array}$ & $\begin{array}{c}\text { Bac } \\
\text { err. }\end{array}$ & $\begin{array}{c}\text { Tot. Sys. } \\
\text { err. }\end{array}$ & Tot. err. \\
\hline $0.001-0.02$ & 0.635 & 0.021 & 0.052 & 0.022 & 0.018 & 0.004 & 0.059 & 0.062 \\
$0.001-0.1$ & 0.445 & 0.020 & 0.031 & 0.015 & 0.007 & 0.002 & 0.035 & 0.037 \\
$0.3-0.8$ & 0.506 & 0.019 & 0.020 & 0.012 & 0.013 & 0.005 & 0.027 & 0.033 \\
\hline
\end{tabular}

Table 4. Summary for the $F_{2}^{\gamma}$ estimated by the TWOGAM generated data for the sample with average $<Q^{2}>=40 \mathrm{GeV}^{2}$.

$F_{2}^{\gamma}$ evolution is shown in Fig.9. The function $a+b \log \left(Q^{2}\right)$ is fitted to the data taking into account the total errors. The previous results from LEP1 study are also taken into account. The parameters obtained by the fit are shown in Table 5.

\begin{tabular}{|c|c|c|c|c|c|}
\hline$x$ range & $a$ & $d a$ & $b$ & $d b$ & $\chi^{2} / n d f$ \\
\hline $0.001-0.02$ & -0.004 & 0.065 & 0.402 & 0.060 & 0.18 \\
\hline $0.001-0.1$ & 0.032 & 0.039 & 0.261 & 0.034 & 0.28 \\
\hline $0.3-0.8$ & -0.021 & 0.088 & 0.333 & 0.058 & 0.39 \\
\hline
\end{tabular}


Table 5. The $Q^{2}$ fit results.

In all three $x$ intervals $\log \left(Q^{2}\right)$ dependence is consistent with linear function.

\section{Conclusions}

The photon hadronic structure function $F_{2}^{\gamma}$ has been studied for the data taken by the DELPHI detector at LEP2 at $\sqrt{s_{e e}}=188-208 \mathrm{GeV}$. The measurements are done in $Q^{2}$ interval from 10 to $260 \mathrm{GeV}^{2}$ and in $x$ range from 0.001 to 0.8 .

Monte Carlo generators used in this analysis give reasonable description of the process.

In the lowest $x$ bin the data are slightly above predictions given by the models (GRV, $\mathrm{SaS}$ ) but close to the prediction of TWOGAM generator which use the similar to the GRV parton density function.

In the $x$ interval $x>0.1$ the data are slightly below the TWOGAM prediction and close to the GRV model prediction. Any of three components used in TWOGAM can be responsible for this excess. Further studies are needed to solve this uncertainty. PHOJET and PYTHIA generators underestimate the low invariant mass region.

Hadronic structure function is estimated as a function of $x$ in three $Q^{2}$ intervals, with mean momentum transfer $\left\langle Q^{2}\right\rangle=19,40$ and $101 \mathrm{GeV}^{2}$. Combining the DELPHI data at $\sqrt{s_{e e}}=91 \mathrm{GeV}$ and $189-208 \mathrm{GeV}$, the evolution of $F_{2}^{\gamma}$ with $Q^{2}$ in the range $0.001<$ $x<0.02,0.001<x<0.1$ and $0.3<x<0.8$ has been shown. From this results the slope $\alpha^{-1} d F_{2}^{\gamma} / d \log \left(Q^{2}\right)$ is found to be consistent with the logarithmic evolution of $F_{2}^{\gamma}$ with $Q^{2}$. The proposed method for the photon structure function study has been demonstrated as being consistent. The model dependent systematic error shown by this procedure is larger than estimated by the unfolding procedure and better reflects our knowledge of the process. Only better modelling which can arise from some new physical constraints on the existing generators can reduce such an error.

\section{Acknowledgements}

We are greatly indebted to our technical collaborators and to the funding agencies for their support in building and operating the DELPHI detector, and to the members of the CERN SL Division for the excellent performance of the LEP collider. 


\section{References}

[1] PLUTO Collab., Ch. Berger et al., Z.Phys. C26, (1984) 353;

Phys. Lett. B149, (1984) 421;

Nucl. Phys. B281, (1987) 356.

[2] JADE Collab., W. Bartel et al., Z.Phys. C24, (1984) 231.

[3] TASSO Collab., H. Althoff et. al., Z.Phys. C31 (1986) 527.

[4] CELLO Coll., H.-J. Behrend et al., Phys. Lett. B126, (1983) 391.

[5] TPC/2 $\gamma$ Coll., A. Aihara et al., Phys. Rev. Lett. 58,(1987) 97;

TPC/2 $\gamma$ Coll., A. Aihara et al., Phys. Rev. 5441 (1985) 736.

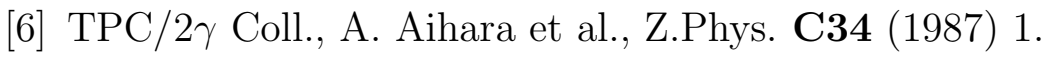

[7] AMY Collab., R. Sasaki et al., Phys. Lett. B252 (1990) 491;

S.K. Sahu et al., Phys. Lett. B346 (1995) 208.

[8] TOPAZ Collab., K. Muramatsu et al., Phys. Lett. B332 (1994) 478.

[9] OPAL Collab., R. Akers et al., Z.Phys. C61 (1994) 199.

[10] DELPHI Coll., P. Abreu et al. Z.Phys. C69 (1996) 223.

[11] OPAL Coll., K. Ackerstaff et al., Z.Phys. C74 (1997) 33.

[12] OPAL Coll., K. Ackerstaff et al., Phys. Lett. B411 (1997) 387.

[13] OPAL Coll., K. Ackerstaff et al., Phys. Lett. B412 (1997) 225.

[14] OPAL Coll., G. Abbiendi et al., Eur. Phys. J. C18 (2000) 15.

[15] L3 Coll., M. Acciarri et al., Phys. Lett. B436 (1998) 403.

[16] L3 Coll., M. Acciarri et al., Phys. Lett. B447 (1999) 147.

[17] L3 Coll., M. Acciarri et al., Phys. Lett. B483 (2000) 373.

[18] ALEPH Coll., R. Barate et al., Phys. Lett. B458 (1999) 152.

[19] OPAL Coll., Jan A. Lauber, Proc. of the 28th Int. Conf on High Energy Physics, World Scientific Publising Co. Pte. Ltd., (1996) 725.

[20] DELPHI Coll., Igor Tyapkin, Proc. of the 28th Int. Conf on High Energy Physics, World Scientific Publising Co. Pte. Ltd., (1996) 729.

[21] S. Nova, A. Olshevsky, T. Todorov, DELPHI-90-35 (unpublished).

[22] M. Drees and R.M.Godbole, J. Phys. G21 (1995) 1559.

[23] L.E. Gordon and J.K. Storrow, Z.Phys. C52 (1992) 307.

[24] M. Gluck, E. Reya and A. Vogt, Phys. Rev. D45 (1992) 3986. 
[25] H. Abramovicz, K. Charchula and A. Levy, Phys. Lett. B269 (1991) 458.

[26] G.A. Schuler and T. Sjostrand, Z. Phys. C68 (1995) 607.

[27] Humboldt University, D-10099 Berlin, FRG.

[28] Workshop on Physics at LEP2 ,CERN 96-01 v2 (1996).

[29] T. Sjöstrand, Comp. Phys. Comm. 82 (1994) 74.

[30] G. Marchesini, B.R. Webber, G. Abbiendi, I.G. Knowles, M.H. Seymour and L. Stanco, Computer Phys. Comm. 67 (1992) 465.

[31] DELPHI Coll., P. Aarnio et al., Nucl. Instr. and Meth., A303 (1991), 233.

[32] DELPHI Coll., P.Abreu et al., Phys.Lett. B342 (1995) 402.

[33] V.N. Baier et al., Phys. Rep. 78 (1981) 293.

[34] H. Plothow-Besch, PDFLIB : A Library of all available Parton Density Functions of the Nucleon, the Pion and the Photon and the corresponding $\alpha_{s}$ calculations, CERN-PPE-92-123.

[35] L. E. Gordon and J.K. Storrow, Z.Phys. C56 (1992) 307.

[36] J.J.Sakurai and D. Schildknecht, Phys. Lett. 40B (1972) 121;

Phys. Lett. 41B (1972) 489.

[37] V. Blobel, Proceedings of the CERN School of Computing, Aiguablava, Spain, (1984), CERN 85-09. 

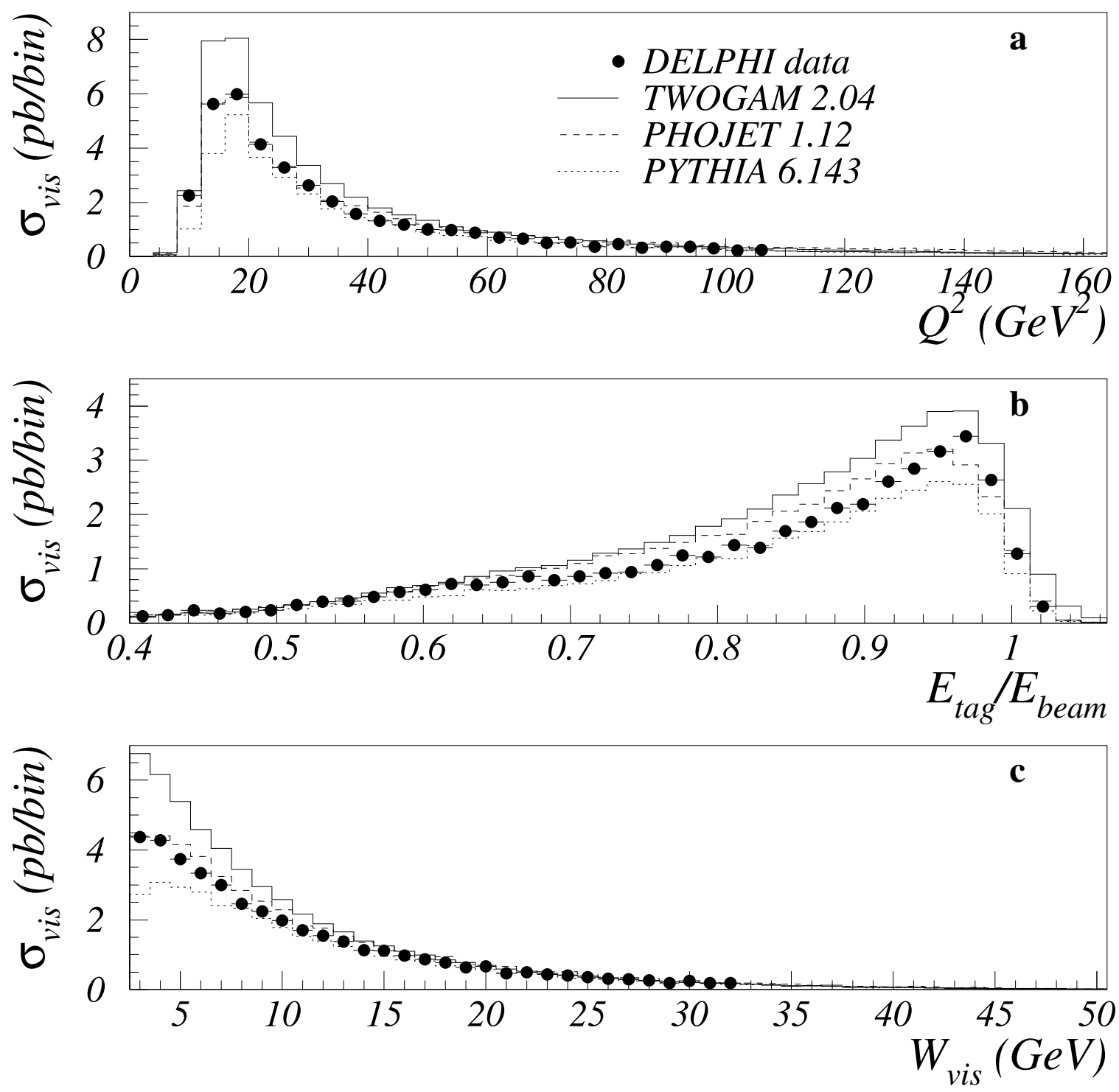

Figure 2: Comparison between data and Monte Carlo predictions for the sample with $<Q^{2}>=40 \mathrm{GeV}^{2}$ : a) $Q^{2}$, b) tagging energy, c) invariant mass. Points are data and the lines show the Monte Carlo predictions from TWOGAM (solid line), PHOJET (dashed line)and PYTHIA (dotted line). 

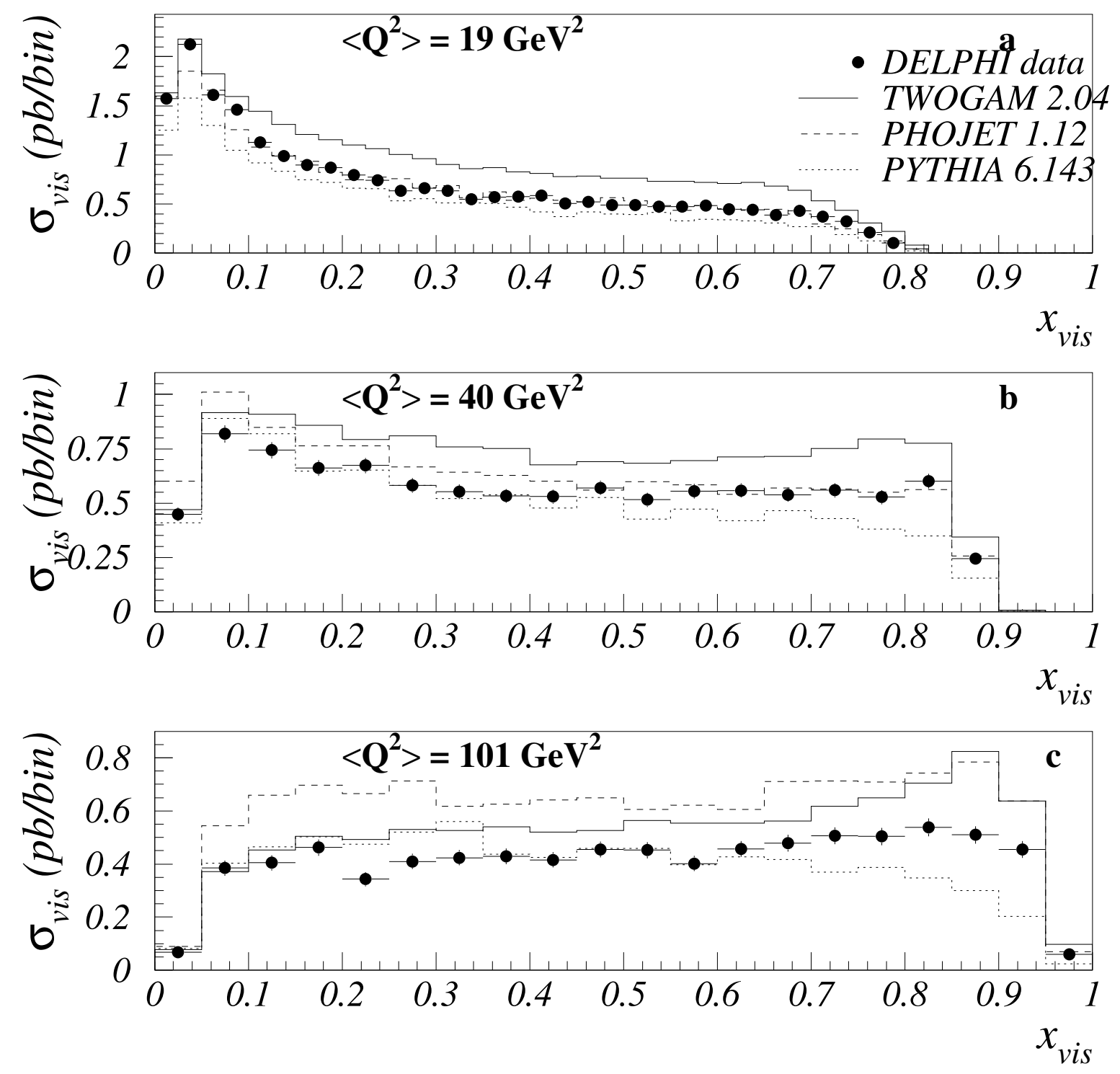

Figure 3: Comparison between data and Monte Carlo predictions for the $x$ visible distributions in diferent $\left\langle Q^{2}>\right.$ regions. Points are data and the lines show the Monte Carlo predictions from TWOGAM (solid line), PHOJET (dashed line)and PYTHIA (dotted line). 

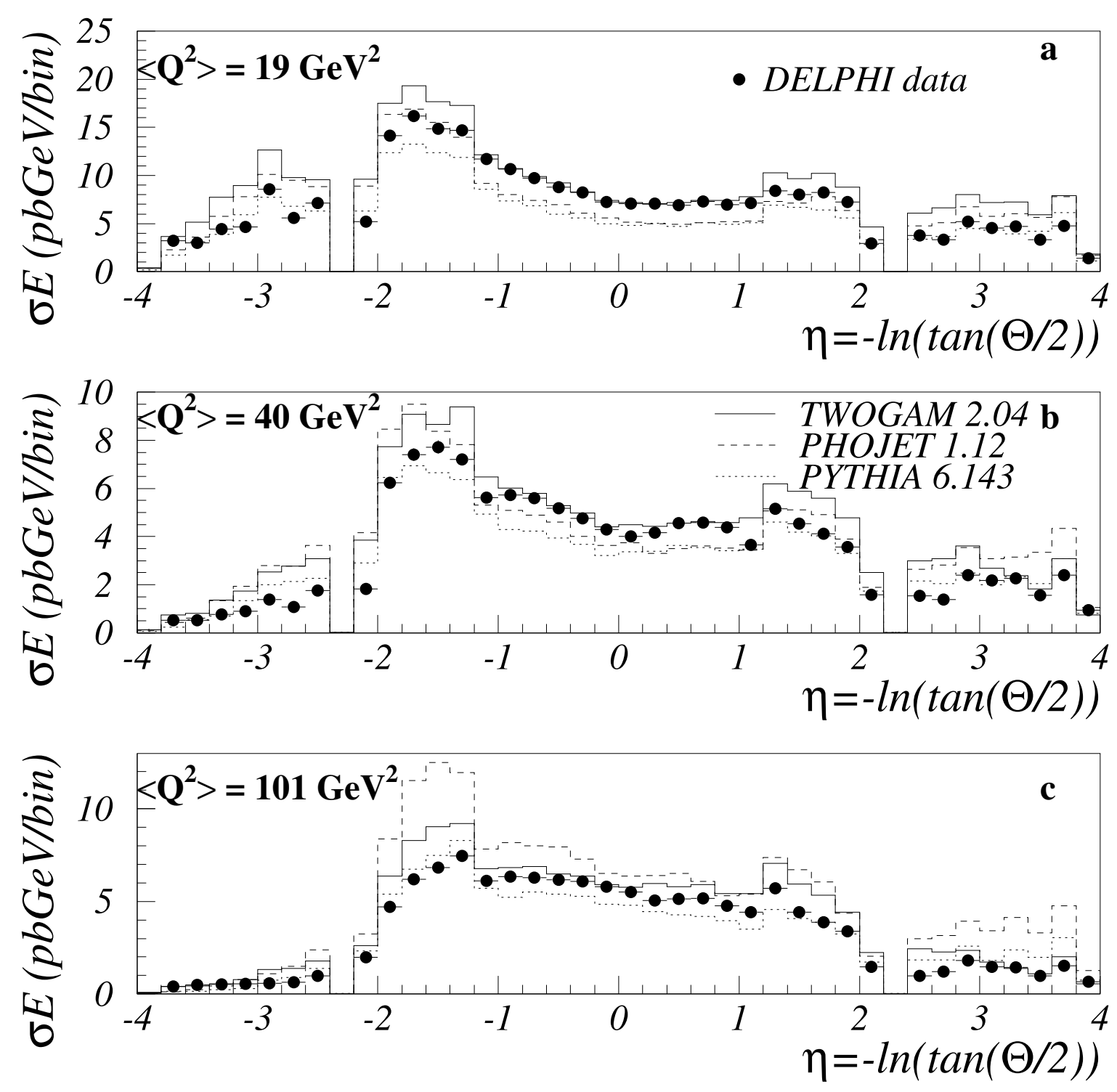

Figure 4: Comparison of hadronic energy flow for data and Monte Carlo prediction in the pseudorapidity scale for the sample with $<Q^{2}>=40 \mathrm{GeV}^{2}$. Points are data and the lines show the Monte Carlo predictions from TWOGAM (solid line), PHOJET (dashed line)and PYTHIA (dotted line). 

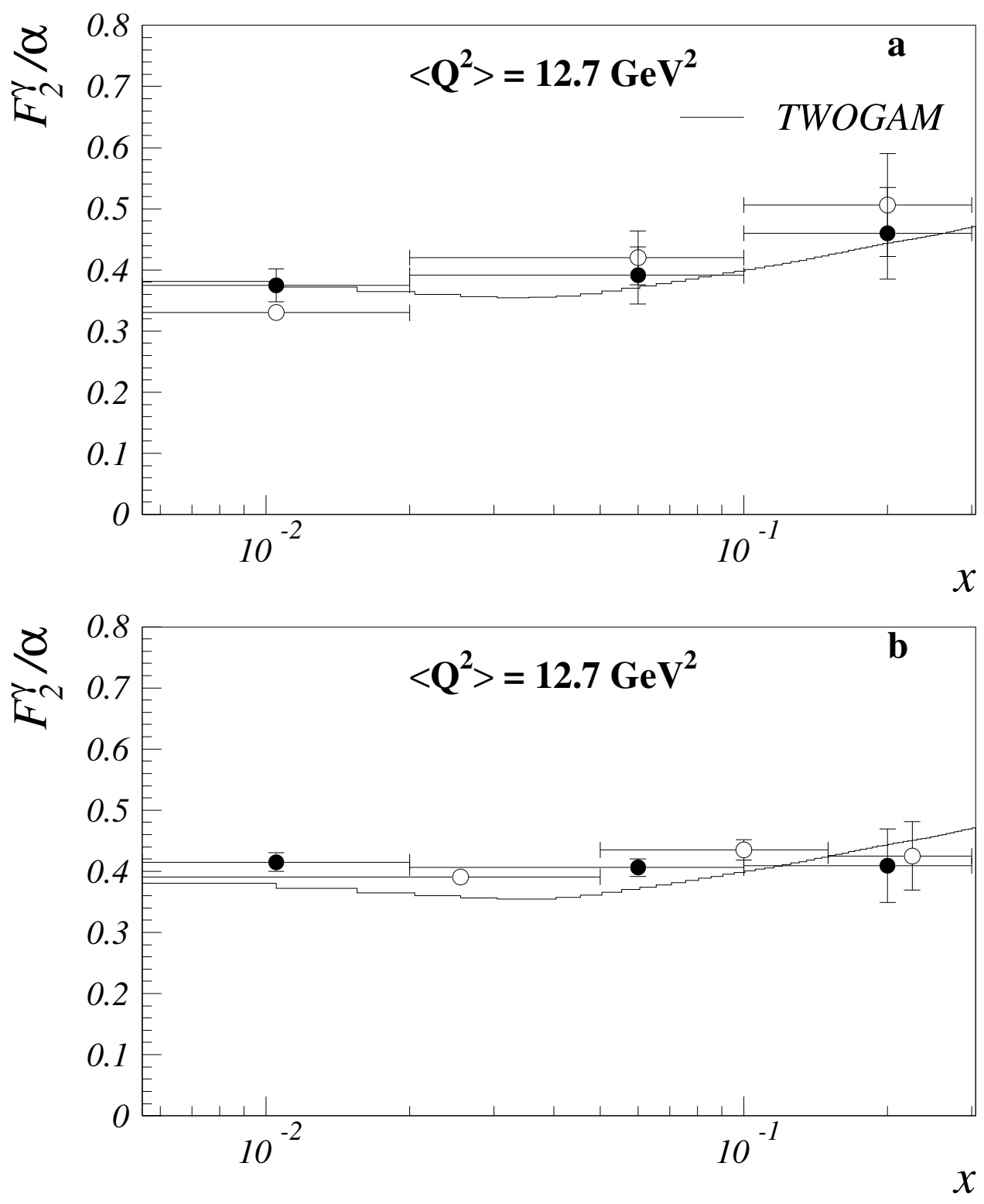

Figure 5: Test of MINUIT based procedure (a) and unfolding (b). a) Open circles- fit with the Monte Carlo sample treated as a sum of three components. Black dots - the complete MINUIT fitting procedure. b) The results of unfolding procedure in different $x$-bins. 


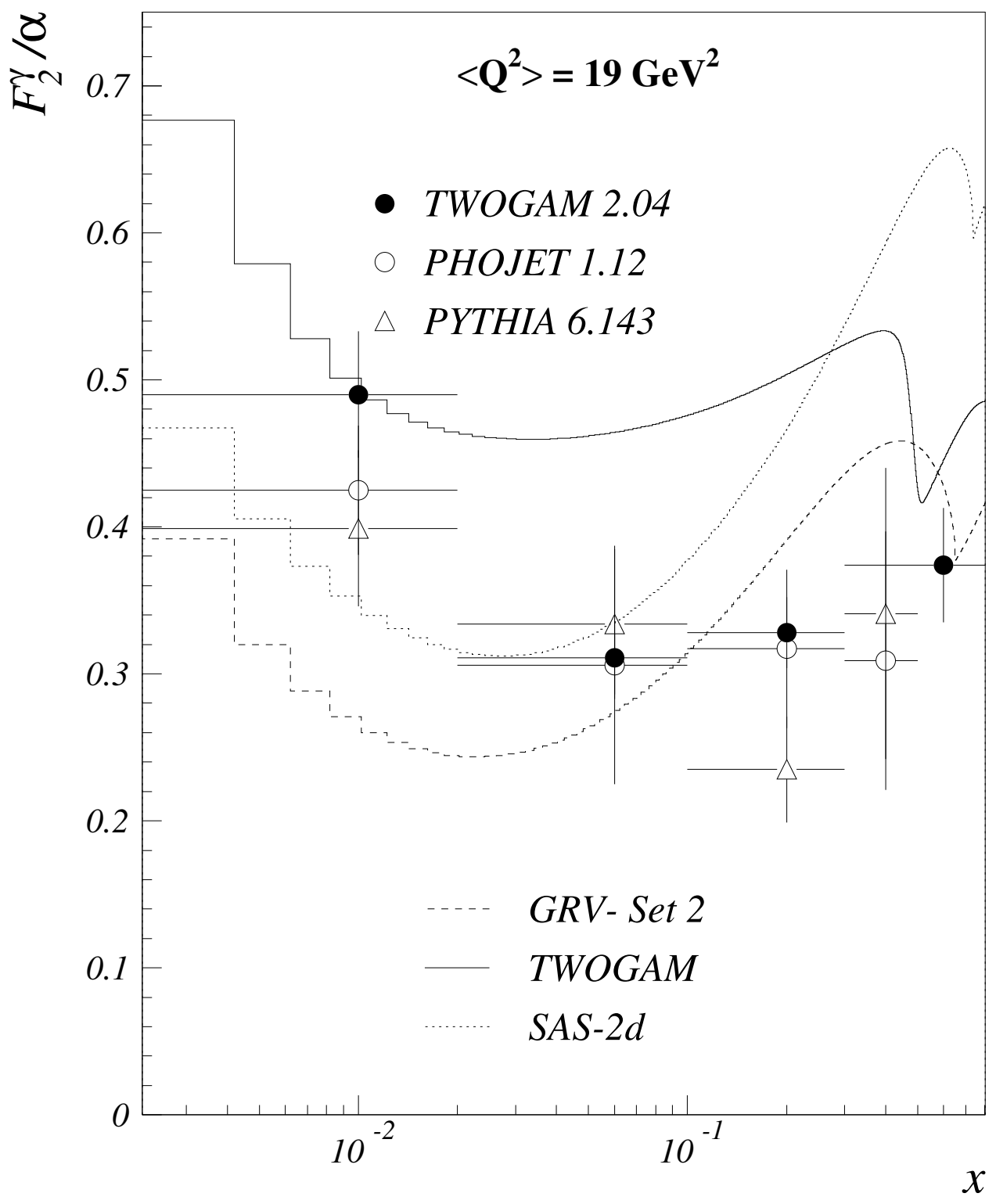

Figure 6: The measured $F_{2}^{\gamma}$ at $\left\langle Q^{2}>=19 \mathrm{GeV}\right.$ as a function of $x$. Error bars show total errors. The data are compared with the predictions of TWOGAM generator, GRV and SaS models. 


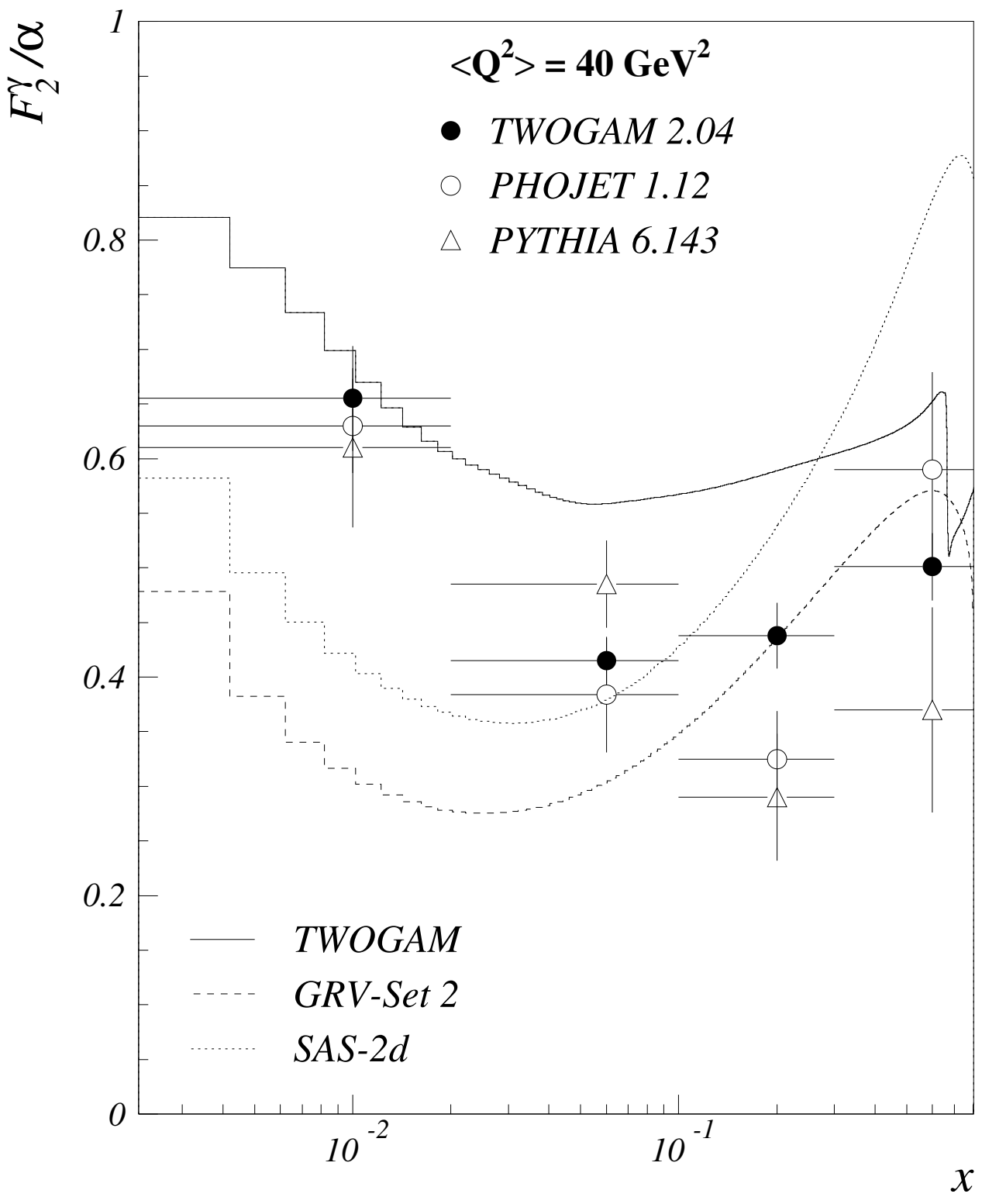

Figure 7: The measured $F_{2}^{\gamma}$ at $\left\langle Q^{2}>=40 \mathrm{GeV}\right.$ as a function of $x$. Error bars show total errors. The data are compared with the predictions of TWOGAM generator, GRV and SaS-2d. 


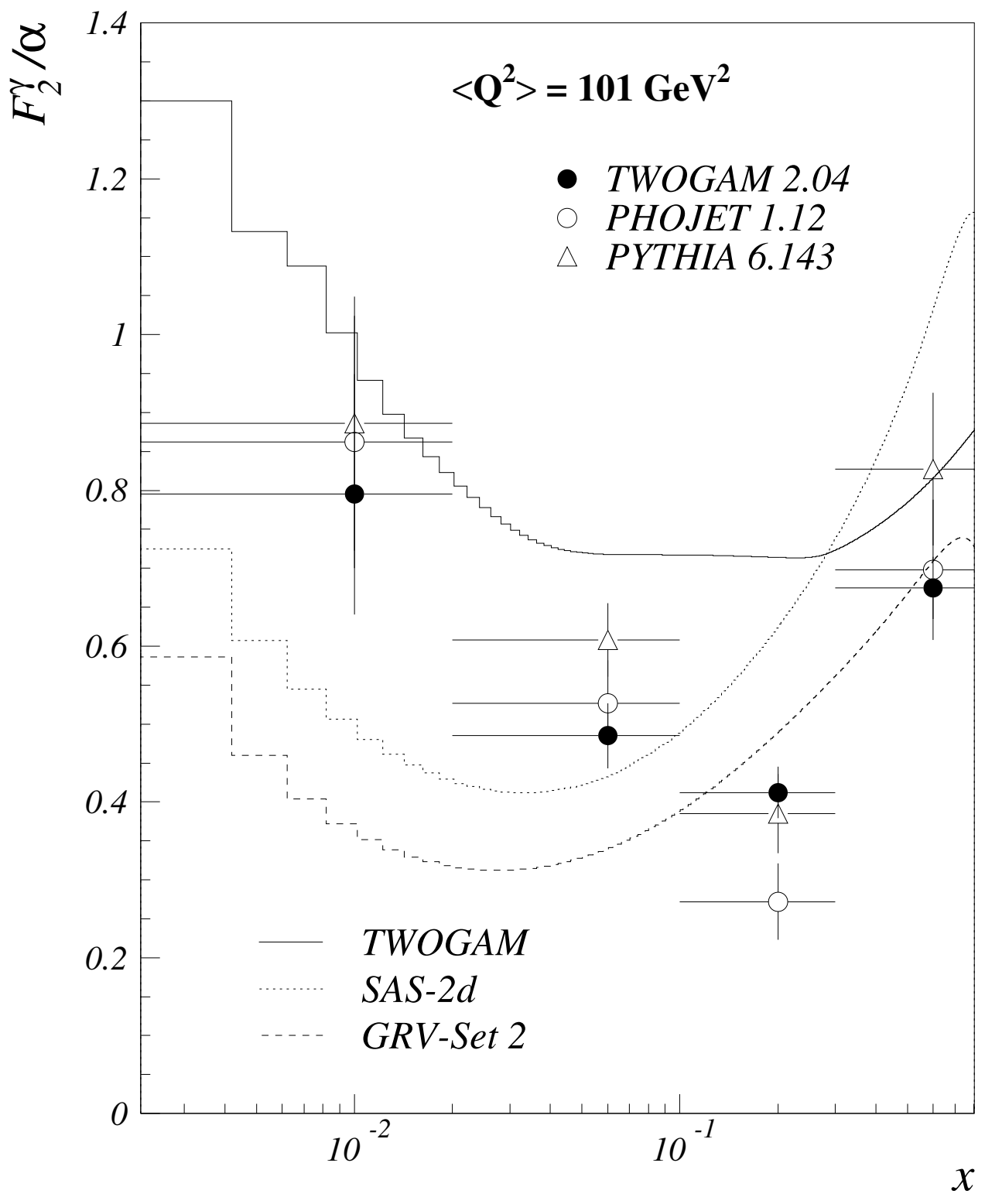

Figure 8: The measured $F_{2}^{\gamma}$ at $<Q^{2}>=101 \mathrm{GeV}^{2}$ as a function of $x$. Error bars show total errors. The data are compared with the predictions of TWOGAM generator, GRV and SaS models. 

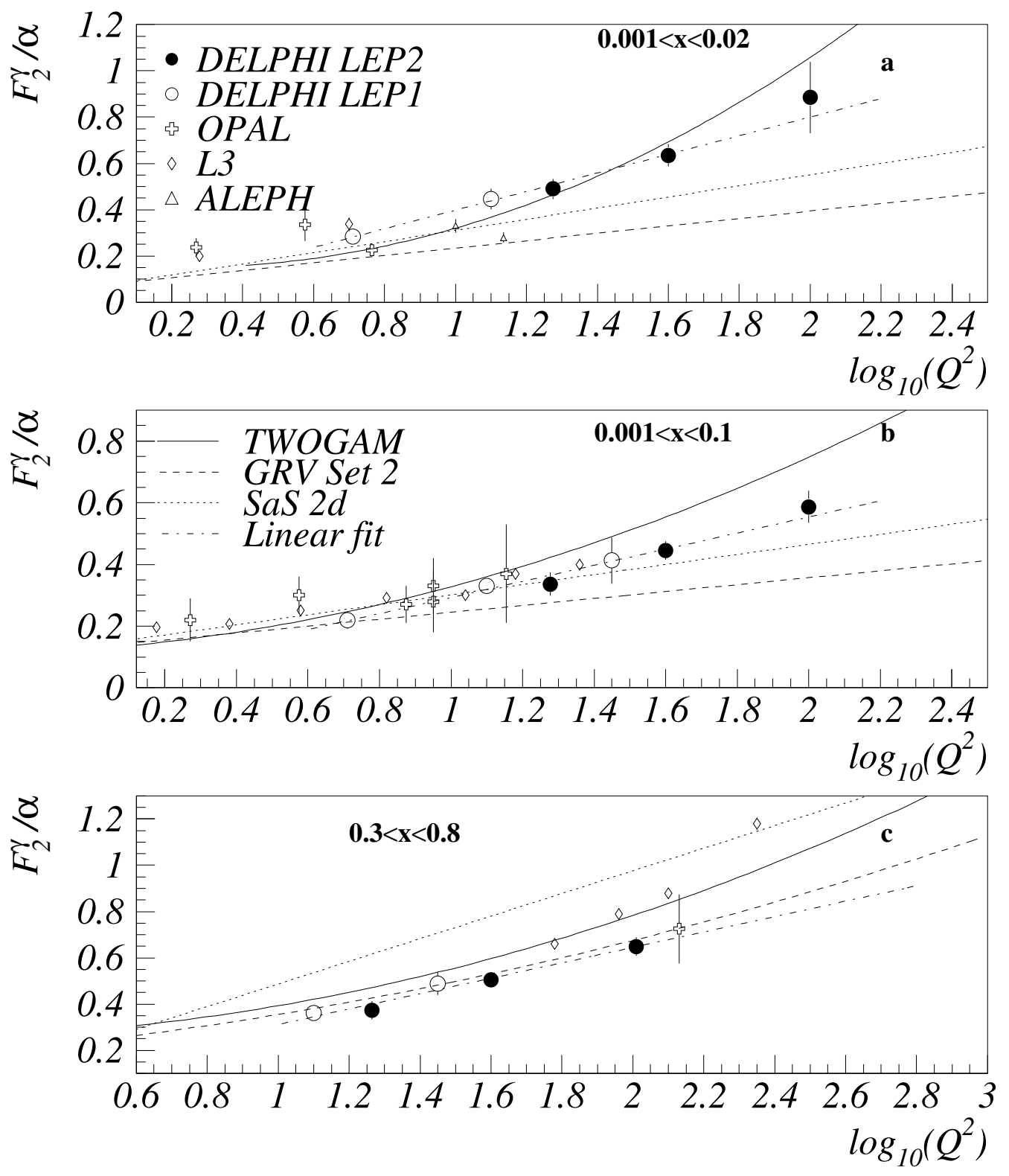

Figure 9: The measured $F_{2}^{\gamma}$ in the different $x$ intervals as a function of $<Q^{2}>$ compared with predictions of TWOGAM generator, GRV and SaS models. The statistical and systematic errors are added in quadrature. 